\title{
Tú vales por lo que eres. El espejismo de un cambio en la relación de los niños/ jóvenes con madres en reclusión
}

\section{Artículo de investigación}

\section{Adris Díaz Fernández}

Universidad de Monterrey, México adris.diaz@udem.edu

\section{Anahí Elizabeth Peña Alba}

Universidad de Monterrey. México anahipeal@hotmail.com

\section{Andrea Amanda Martínez Pérez}

Hult International Business School, Boston, MA, Estados Unidos andrea.amp9@hotmail.com

\section{Ana Lucía Guerra Martínez}

Educativo Liceo de Monterrey, Monterrey, México annie.g.mtz.4@gmail.com

$-$

Recibido: 9 de octubre de 2019

Aprobado: 24 de febrero de 2020

Cómo citar este artículo: Díaz Fernández, Adris; Peña Alba, Anahí; Martínez Pérez Andrea; Guerra Martínez, Ana (2021). Tú vales por lo que eres. El espejismo de un cambio en la relación de los niños/jóvenes con madres en rec/usión. Calle 14: revista de investigación en el campo del arte 16(29). pp. 68-77.

https://doi.org/10.14483/21450706.17402

\section{(2) (1)}

https://creativecommons.org/licenses/by/4.0/deed.es 


$$
\begin{array}{lllllll}
0 & 0 & 0 & 0 & 0 & 0 & 0 \\
0 & 0 & 0 & 0 & 0
\end{array}
$$

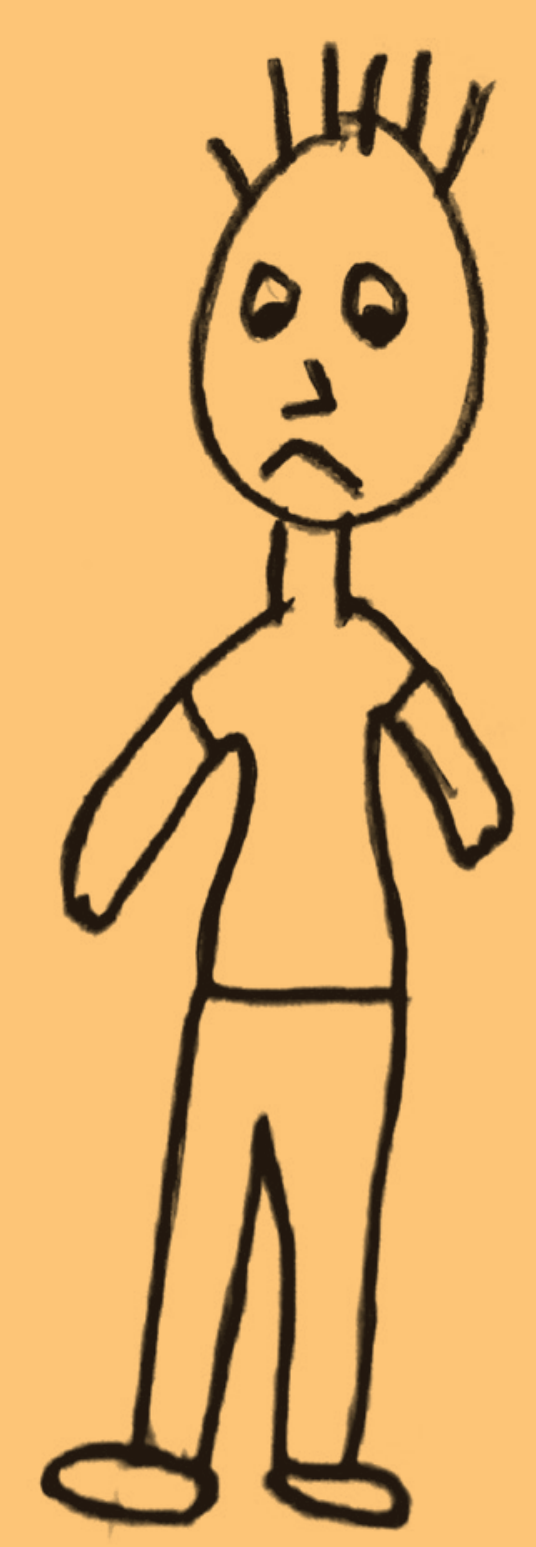


Tú vales por lo que eres. El espejismo de un cambio en la relación de los niños/jóvenes con madres en reclusión

\begin{abstract}
Resumen
El trabajo tiene como propósito identificar la relación madre-hijo-tutor para mejorar el vínculo familiar. El estudio estuvo dirigido a niños/jóvenes de 8 a 15 años de edad cuyas madres se encuentran presas, en Nuevo León, México. Investigación cualitativa que utilizó las pruebas proyectivas "La familia" y "Niño bajo la lluvia" para identificar los deseos íntimos e inconscientes de los niños/jóvenes y sus necesidades afectivas. Se estableció un plan de acción participativo a través de la expresión plástica y una evaluación de su composición y sus efectos. Se concluyó que no existe un vínculo afectivo entre niños-madre (presas)-tutor, dejándoles a los niños/ jóvenes miedos, carencias, inseguridad y baja autoestima. La pesquisa evidencia cómo el arte es una vía ideal para redescubrir situaciones de apego, sueños y desconciertos en situaciones de abandono, de exclusión y un medio eficaz para lograr cambio de actitud y comportamiento.
\end{abstract}

\title{
Palabras claves
}

Arte; conducta de apego; estado emocional; relación familiar; grupo vulnerable

You Are Worthy for What You Are. The Mirage of a Change in the Relationship of Children/Young People With their Inmate Mothers

\begin{abstract}
The purpose of this article is to identify the mother-child-guardian relationship to improve family bonds. The study was aimed at children/young people 8 to 15 years old whose mothers are imprisoned in Nuevo León, Mexico. This qualitative research used the projective tests "The family" and "Child in the rain" to identify the intimate and unconscious desires of children/young people and their affective needs. A participatory action plan was established through plastic art expressions and an evaluation of their creation and effects. The study concluded that there is no affective bond between children-mother (inmates)-tutor, leaving children/young people with fear, unfulfilled needs, insecurity and low self-esteem. The research shows how art is an ideal way to rediscover situations of attachment, dreams and bewilderment in situations of abandonment, exclusion, as well as an effective means to achieve a change in attitude and behavior.
\end{abstract}

\section{Keywords}

Art; attachment behavior; emotional state; family relationship; vulnerable group

Vous êtes précieux pour ce que vous êtes. Le mirage d'un changement dans la relation des enfants / jeunes avec leurs mères détenues

\section{Résumé}

Le but de cet article est d'identifier la relation mère-enfant-tuteur pour améliorer les liens familiaux. L'étude était destinée aux enfants / jeunes de 8 à 15 ans dont les mères sont incarcérées à Nuevo León, au Mexique. Cette recherche qualitative a utilisé les tests projectifs «La famille» et «Enfant sous la pluie» pour identifier les désirs intimes et inconscients des enfants / jeunes et leurs besoins affectifs. Un plan d'action participatif a été établi à travers les expressions des arts plastiques et une évaluation de leur création et de leurs effets. L'étude a conclu qu'il n'y a pas de lien affectif entre les enfants-mère (détenus)-tuteur, et les enfants / jeunes finissent avec des sentiments de peur, manque, insécurité et une faible estime de soi. La recherche montre comment l'art est un moyen idéal pour redécouvrir des situations d'attachement, de rêve et de perplexité dans des situations d'abandon, d'exclusion, et aussi un moyen efficace de changer d'attitude et de comportement. 


\section{Mots clés}

Art ; comportement d'attachement ; état émotionnel ; relation familiale et groupe vulnérable

Você vale pelo o que é. A miragem de uma mudança na relação de crianças/jovens com mães presas.

\section{Resumo}

O trabalho tem como propósito identificar a relação mãe-filho-tutor para melhorar o vínculo familiar. 0 estudo foi direcionado à crianças/jovens de 8 a 15 anos de idade cujas mães se encontram presas, em Novo Leão, México. Investigação qualitativa que se utilizou dos testes projetivos "La familia" e "Niño bajo la lluvia" para identificar os desejos íntimos e inconscientes das crianças/jovens e suas necessidades afetivas. Se estabeleceu um plano de ação participativa através da expressão plástica e uma avaliação de sua composição e seus efeitos. Se concluiu que não existe um vínculo afetivo entre crianças - mães (presas)-tutor, deixando medos, carências, insegurança e baixa autoestima às crianças/ jovens. A pesquisa evidencia como a arte é uma via ideal para redescobrir situações de apego, sonhos e desorientações em situações de abandono e exclusão e, um meio eficaz para conseguir uma mudança de atitude e de comportamento.

\section{Palavras chave}

Arte; conduta de apego; estado emocional; relação familiar; grupo vulnerável

Kam kangui llapa ministidu kaipi kawachii musukunata/ guaguakunata warmikuna ukullapi kaskakunata

\section{Maillallachiska}

Pangapi mailla kilkaspa parlakumi imasami kaugsankuna guaguakuna, musukuna, sapalla. Atun Ilakikunaua paikunapa mamakuna kanguna carcilpi chimanda wambrakunapas. Chillapi kankuna. Paikunata mana lisinsiankuna llugsingapa, pisikumi, achka alli munaspa suma kullaspa iachachinga kaugsaita munangapa. Kunaura sug ruraikunaua munanaku ninga paikunapas munaspa pudinkunami kanga nukanchisina allilla runakuna.

\section{Rimangapa ministidukuna}

Arte ruraikuna; llapa munag; imasami kausakuni; munanakuspa kausasunchi; llapa ministidukunaua kausai 


\section{Introducción}

El presente estudio se incluye dentro de la investigación titulada "Madre, hijo, tutor: Efectividad de un proyecto de intervención pedagógico enfocado en lo artístico y cultural, para la convivencia sana y adaptación familiar de los niños/jóvenes, con madres presas dentro del penal de Topo Chico", pesquisa realizada en el instituto Renace A.B.P., en Monterrey, México. "Tú vales por lo que eres. El espejismo de un cambio en la relación niños/ jóvenes con madres en reclusión" tiene como objetivo valorar la relación de afecto madre-hijo-tutor, de los niños/jóvenes de 8 a 15 años del programa Renace niño 2 del instituto Renace A.B.P., mediante pruebas proyectivas, atención personalizada y el diseño de acciones artísticas que favorezca el hallazgo y/o elucidación de emociones y sentimientos ocultos, propiciando su desarrollo creativo y el vínculo afectivo madre-hijo-tutor.

Es de hacer notar que el estatus emocional de los niños/jóvenes de las madres reclusas es un tema relevante y de preocupación en la actualidad. La separación de los niños/jóvenes de sus madres reclusas trae consecuencias en su comportamiento y limita su inclusión en la sociedad. En el artículo se señala como factores importantes la relación madre-hijo-tutor y el papel del arte como medio para conocer y lograr un vínculo afectivo de los hijos con su madre y una positiva inserción de los jóvenes a la sociedad.

En México se torna preocupante la situación de los reclusos. El INEGI (Instituto Nacional de Estadística, Geografía e Informática) señala que para el cierre del 2016 se registró un total de 188.262 personas privadas de la libertad en los centros penitenciarios de las entidades federativas, de las cuales $95 \%$ eran hombres y el $5 \%$ restante, mujeres; estas son minoría en la cadena delictiva, pero su presencia en las prisiones estatales ha aumentado en los últimos años (56\% de 2010 a 2015) (2017, p. 11). Información que marca un punto neurálgico y de desasosiego con relación a la formación de sus hijos.

Esta situación se ha convertido en un compromiso para algunas instituciones y organizaciones en el país, que se preocupan por atender a los hijos de reclusos, un ejemplo es la institución Renace A.B.P., en el municipio de Monterrey en el estado de Nuevo León, que por más de 23 años ha trabajado por la mejora continua de la justicia penal en México. Dentro de sus programas de ayuda se encuentra Renace Mujer, enfocado en darles un proyecto de vida a las mujeres reclusas (Renace,
2016). Otro de los programas institucionales es Renace Niño que tiene como finalidad brindar atención social a los hijos de madres y padres de familia en reclusión con talleres de actividades culturales, recreativas y de esparcimiento (Aporta, 2017).

En este escenario se desarrolla la investigación. La idea de situar al arte como eje central del trabajo está asociado a las opciones que es capaz de ofrecer, tales como ser detonador de sentimientos y sueños, puente para resolución de problemas, identificador de necesidades y de acciones colectivas. El arte tiene en el ser humano consecuencias positivas que fortalecen su aprendizaje y desarrollan en él conexiones que tal vez sólo el lenguaje artístico puede propiciar. Puede servir de ejercicio terapéutico para comprender mediante la imaginación qué es lo que sucede por un lado, y "estimula las capacidades propias del individuo, desarrolla su creatividad y expresión individual como medio de lograr satisfacción y mejora personal" (López, 2004, p.3).

\section{Metodología}

La Investigación Acción Participación (IAP) es el método de estudio empleado. Se acudió a la observación estructurada, pruebas proyectivas, análisis de las obras artísticas, la entrevista y la convivencia como métodos de recogida de información. Con una población de 25 niños/jóvenes entre 4 y 15 años de edad de madres presas y una muestra de 12 niños/jóvenes quienes se encuentran en el rango de 8 a 15 años de edad que actualmente viven con sus abuelas (tutor). Dicha muestra fue seleccionada por interés de la institución Renace A.B.P. dada la vulnerabilidad de esas edades.

\section{Desarrollo}

El arte es trascendental en la formación, ya que fomenta el proceso de la expresión creativa natural que todo ser trae consigo, y estimula tanto las cualidades como los valores sociales, morales y la autoestima. Simultáneamente tiene la intención de introducir al hombre en el seductor mundo de la construcción y apreciación artística, y en la expresión, elementos que aportan al espíritu creativo y social de todo ser humano. De esta forma se puede deducir que el arte no es exclusivo de los artistas, por el contrario, es una condición de expresión que tienen en común todos los seres humanos, es un referente para descubrir situaciones y estados de ánimos que van más allá de todo aquello 
que podemos expresar sin miedos a hacer cuestionado, subvalorado o poco entendido. "El arte permite proyectar conflictos internos y, por lo tanto, ofrece la posibilidad de poder resolverlos" (López, 2004, p. 3).

En los grupos vulnerables es necesario crear espacios de confianza y respeto, ya que la seguridad y la autoestima difícilmente se generan en ambientes hostiles. Sin lugar a dudas, el espacio de convivencia genera condiciones que permiten un mayor o menor grado de libertad, un determinado sentimiento de apego, un estado emocional y una relación familiar débil, como es el caso de los hijos de las madres presas.

El apego, elemento clave de la relación, es el lazo recíproco y duradero entre dos personas, buscando contribuir a la calidad de la relación (Feldman, Papalia, y Wedkos Olds, 2009). El estado de ánimo se puede definir como una emoción generalizada y persistente que da un panorama de la percepción del mundo (APA, 1995) (en Balladares y Saiz, 2015). De acuerdo a las condiciones que propician los cuidadores junto con el ambiente se puede inferir que el vínculo es distinto en cada situación, en algunos casos predominan las relaciones de seguridad y confianza, mientras que en otros casos se propician las relaciones de inseguridad y ansiedad (Sánchez, 2012). Ekman (2012) afirma que los estados de ánimo no se pueden apreciar ni en la cara ni en la voz. Por su causa difusa se desarrollan emociones concretas, las cuales llevan a percibir e interpretar el entorno de una manera en específico al igual que responder a las situaciones de manera predeterminada (Balladares y Saiz, 2015).

Los estados de ánimo pueden involucrar tanto el afecto negativo como el positivo, volviendo a éste bidimensional. El afecto positivo (AP) demuestra el nivel hasta el cual una persona se siente activa, entusiasta, con energía y con una participación gratificante. Mientras que el afecto negativo (AN) refleja una dimensión general de participación desagradable que incluye distintos estados emocionales aversivos como disgusto, miedo, ira, culpa y nerviosismo (Balladares y Saiz, 2015).

Según Newmark (2002) existen cinco necesidades básicas las cuales los adultos deben satisfacer en el marco familiar, con el fin de que los niños/jóvenes tengan un desarrollo emocional apropiado y estén bien consigo mismos. Estas necesidades son: a) sentirse respetados; b) importantes; c) aceptados; d) incluidos; e) seguros.
La familia es más que la suma de sus miembros, ya que cada uno influye en el todo y este impacta en cada uno (Macías, Marín, y Cantillo, 2004). El sociólogo Páez (1984) (en Macías, Marín, y Cantillo, 2004) define a la familia como núcleo de la sociedad en el cual el individuo aprende creencias, valores y normas a interactuar con los medios que lo rodean y lo prepara para enfrentarse a la sociedad. Además, se encarga de la socialización durante la vida de las personas, donde aprenden a ser ellos mismos, asumiendo diferentes roles y estatus adquiridos.

La calidad de relación con la familia organiza sus modelos cognitivos internos y las relaciones con los demás, ya que cuando hay una falta de comunicación con los padres, esta se ve asociada a las dificultades con los amigos, llevando a un malestar físico y psicológico, lo cual puede conducir a consumos de adicciones en edades muy tempranas (Cobos, 2008). Por lo cual algunas de las funciones esenciales que corresponden a la familia son: la socialización, el afecto, el cuidado, el estatus, entre otros (Medigraphic, 2005).

Mantener relación con la madre es algo esencial, más aún si ésta era débil antes de entrar al reclusorio. Es una relación positiva en el caso que tanto los niños/ jóvenes como las madres buscan continuar viéndose y manteniendo contacto unos con otros. Adalist-Estrin (2003) menciona algunos beneficios que se derivan del contacto con los presos. Uno es que ellos pueden ser una influencia positiva en la vida y crianza de los hijos, ya que no porque hayan fracasado como ciudadanos significa que no pueden tener éxito como madres; estar en la cárcel puede ser una oportunidad de mejorar.

Adalist-Estrin (2015) alude que es muy importante tener siempre en cuenta que tanto los niños/jóvenes como los adolescentes reaccionan de diferente manera al encarcelamiento de alguno de sus padres y estas diferencias dependen de su edad, personalidad, circunstancias familiares, situaciones estresantes a su alrededor, detalles de delito y del encarcelamiento, así como recursos profesionales disponibles.

El rol que debe tener un tutor o padre sustituto es "ser el puente" con los padres biológicos del niño/joven, es decir, mantenerlos siempre informados de lo que está sucediendo, del comportamiento del niño/joven, de cómo se va desarrollando tanto social, académica y emocionalmente, entre otros componentes de los que los padres deben estar al tanto para que sus hijos se sientan más queridos (Raffo, 2009). 


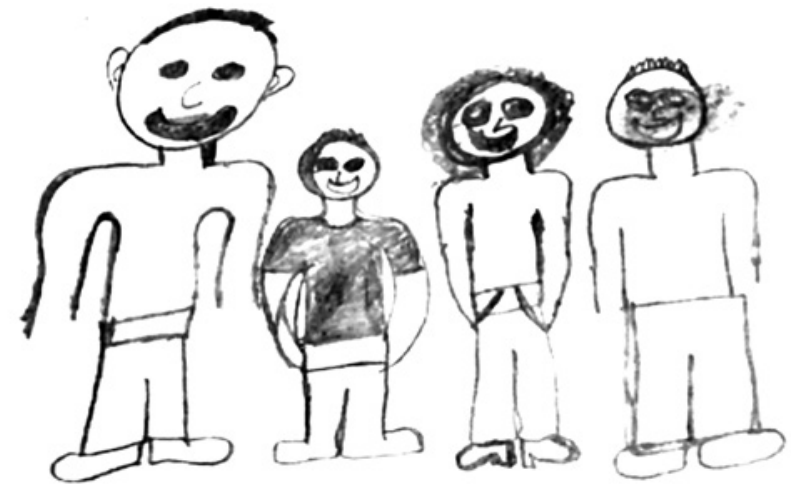

Imagen 1. Prueba proyectiva "La Familia", febrero 2017.

\section{Análisis y discusión de los resultados}

Para evaluar el vínculo que tienen los niños/jóvenes con su propia familia, se aplicaron las pruebas proyectivas, "La familia" y "Niño bajo la lluvia". Entendemos el vínculo como el impulso o la relación que se caracteriza por buscar y preservar la proximidad, en este caso la familiar.

Al analizar los resultados, se pudo comprobar aquellos sentimientos que de forma consciente los muchachos no habían podido demostrar. En ambas pruebas se denotó que niños y jóvenes presentan aspectos de timidez, ansiedad, depresión, retraimiento con la sociedad, no se sienten parte de la familia, son hostiles ante el mundo, presentan dificultad para proyectarse al futuro, afloran sentimientos de culpabilidad, violencia, se consideran incapaces de defenderse; contaban con baja autoestima, incertidumbre, dificultad para conectarse con los demás, culpabilidad, temor a la madre, tienen conflictos sin resolver y preocupaciones por relaciones sociales, viven dominados por las reglas y sufren de falta de decisión.

La observación estructurada además aportó datos interesantes, tales como: entre los niños/jóvenes y entre los tutores no había síntomas de convivencias, a pesar de simpatizar por vivir en realidades parecidas; de igual manera, no se lograba un vínculo de gozo, de diversión entre niños/jóvenes y tutor, sino de protección, autoridad e imposición, incidiendo de manera directa en el pensar y accionar del niño/joven, situación que provocaba emociones de tristeza, miedo e incertidumbre al estar entre personas desconocidas.

A partir de estos resultados se decidió trabajar estas problemáticas a través del arte. Para su desarrollo se
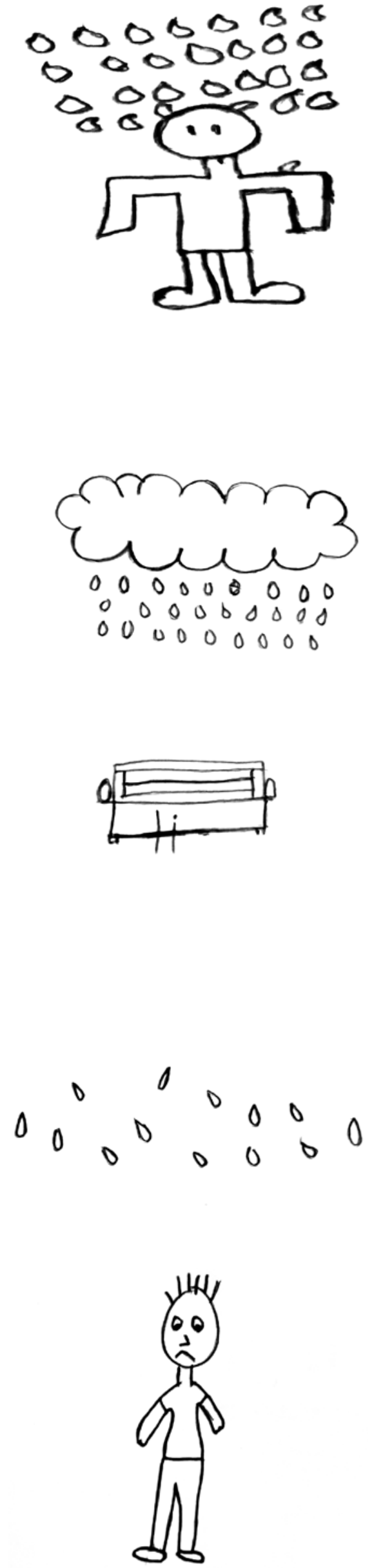

Imagen 2, 3 y 4. Prueba proyectiva "Niño bajo la lluvia", febrero 2017. 

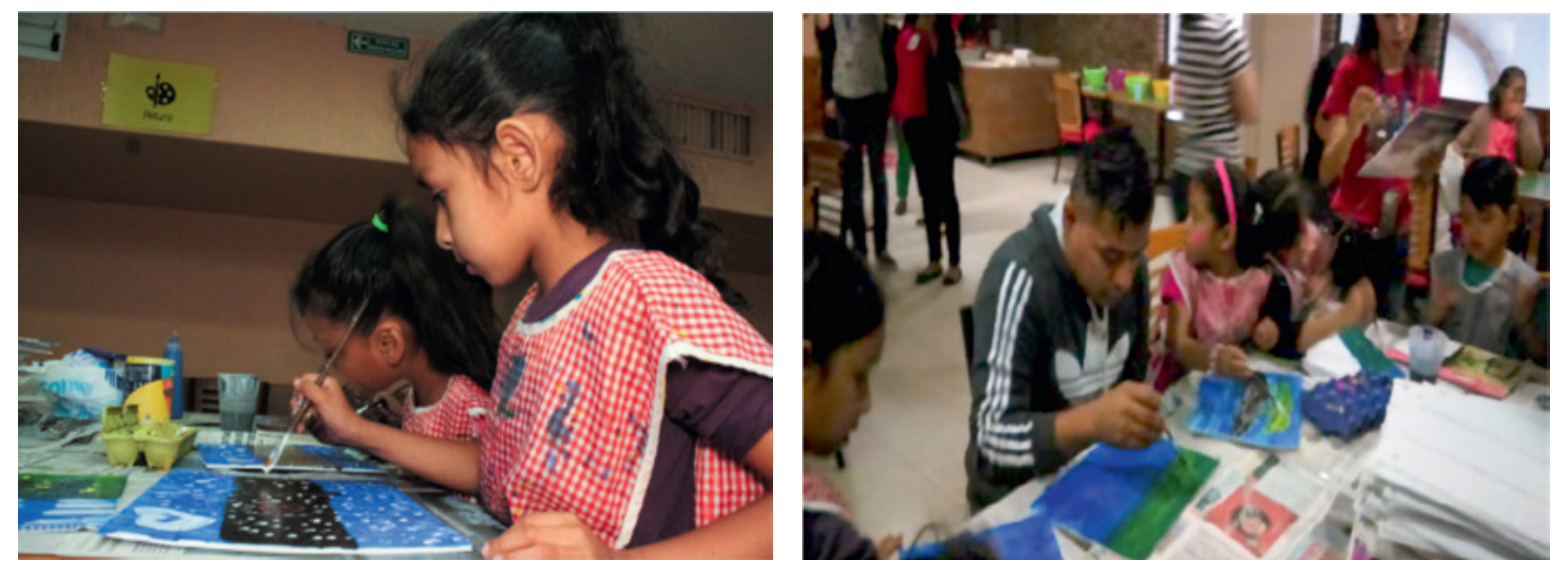

Imagen 5. Taller de pintura bajo la asesoría de la Fundación Kawoq 13:20, abril, 2017.

contó con el apoyo de promotores, como la Fundación Kawoq 13:20 y el Colectivo Caminando en Mi Barrio.

El taller de plástica se convirtió en una experiencia de aprendizaje experiencial donde predominó una relación dinámica que permitió la exploración, el reconocer y el apreciar al otro, trabajando de manera conjunta en el reconocimiento de sí mismo y del otro. En definitiva, es importante para "el individuo que pueda identificarse con lo que hace, conocerse, saber cómo piensa, expresar lo que siente y ser partícipe del mundo que lo rodea, De esta forma, puede desarrollar aptitudes positivas hacia sí mismo y hacia los demás" (López, 2004, p. 3).

El Taller "Haz tú corazón" con la técnica de Trencadis, tenía como objetivo realizar un corazón, forrado de piezas de mosaico, cortado y unido a modo de un rompecabezas, en él cada participante debía escribir el nombre de la persona que más quiere. Se expusieron nombres de tutores, primos, hermanos y no de las madres, quedando demostrado que las figuras más importantes de su vida eran aquellas que estaban a cargo de su cuidado y protección.

El espacio de convivencia se convirtió en un lugar de encuentro, de anécdotas y experiencias, como no había sucedido nunca antes, un sitio en donde afloraban sentimientos y emociones que, independientemente de tornarse triste por los recuerdos y situación existente en su vida familiar, fue muy liberador para sus participantes, porque todos de una forma u otra compartían la misma realidad.

Con la finalidad de contribuir a elevar su autoestima, las obras fueron expuestas en "Gira soy Meshico", exhibición presentada en el parque fundidora en Monterrey.
Esta acción fue un ingrediente decisivo para el esfuerzo de ayudar a elevar la autoestima de los niños/jóvenes. Se fortaleció a su vez el vínculo con las tutoras, logrando entre ellos un reconocimiento al talento, una mayor libertad de expresión y actuación colaborativa basada en una alianza afectiva.

Las pruebas proyectivas y las acciones artísticas revelaron que los niños/jóvenes cuyas madres se encuentran presas son personas con carencias afectivas en relación a la madre, situación que incide de manera directa en su modo de actuar y sentir, llevándolos a mostrar en ocasiones ira, tristeza, miedo; y que el arte es una vía ideal para descubrir y exponer sentimientos, emociones, además de canalizarlos de una manera sana y creativa.

La información recopilada sirvió de pauta para trabajar el vínculo afectivo que el niño/joven necesita, primero con su tutora, puesto que actualmente es con la que más convive y posteriormente poder trabajar ese vínculo con la madre, sin dejar atrás la relación tutora-madre. Resultados que ayudaron a proyectar nuevas acciones al programa "Renace mujer y renace niño" del instituto Renace A.B.P.

\section{Conclusión}

"Hay que dar al niño hombros para que sustente el peso que la vida le eche encima, no peso ajeno que oprima sus hombros". José Martí, Obras Completas, tomo 8, p. 291

La falta de apego del niño/joven con su madre presa genera un afecto inseguro y agresivo, la sensación de abandono incentiva a que estos niños/jóvenes no 


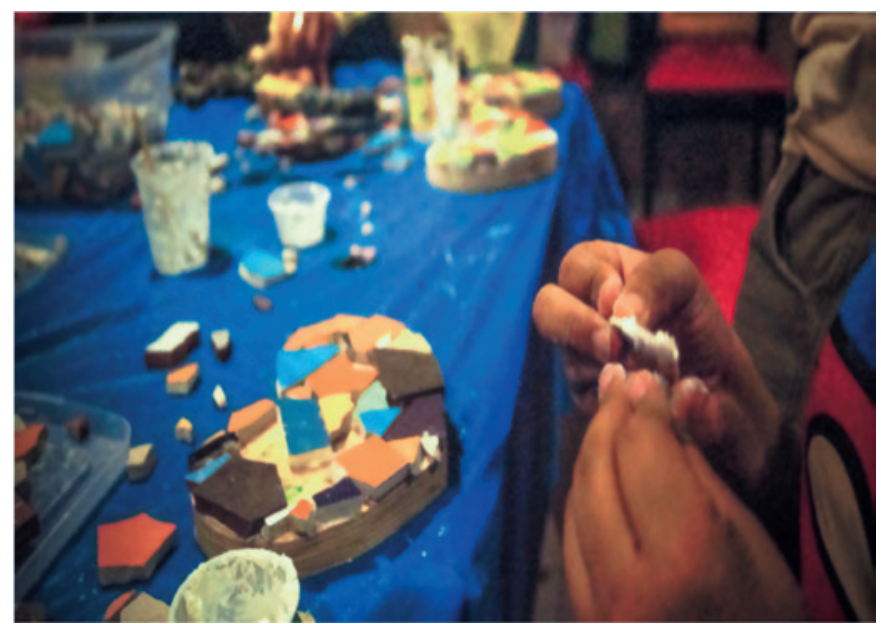

Imagen 6. Taller de Trencadís bajo la asesoría del colectivo Caminando en Mi Barrio, abril, 2017.

mantengan relaciones saludables y seguras. Queda en evidencia que estos niños/jóvenes necesitan elevar su autoestima, mejorar su relación con su madre, tutor -abuelas a cargo de su cuidado-, familiares e iguales, y es el arte una vía importante para lograr estos propósitos.

El vínculo, afectivo o no, con la madre - presa- concederá en los niños/jóvenes conductas, cualidades e inclinaciones que estarán visibles durante toda su vida. Son las familias sus raíces; los niños/jóvenes no precisan conocer cómo maniobrar la ausencia de su madre, ellos lo que necesitan es un vínculo, una relación sólida y profunda que les asegure confianza, seguridad y protección de las lesiones que le provoca el entorno que les rodea.

Estos niños/jóvenes, cuyas madres están presas, son un grupo vulnerable que, a diferencia de otras poblaciones, están abiertos y motivados a experiencias que los nutran para poder enfrentar su vida cotidiana y lograr una relación afectiva, expresiva y cálida.

Trabajar con los sentimientos a través de las manifestaciones artísticas es la clave para expresar emociones vividas, situaciones complejas y deseos que son difíciles de expresar de manera verbal, por lo que el arte constituye un medio de expresión y comunicación efectivo que beneficia el desarrollo sano e integral de los implicados y su integración a la sociedad de manera saludable. Ayuda, además, a valorar de una manera consciente la naturaleza de los miedos, de las desesperanzas, de las tristezas y enojos, convocándolos a vivir una vida plena en armonía con su entorno, con su espacio de intimidad, a crecer de forma sana y a elevar su autoestima, pero sobre todo les ayuda a afrontar las dificultades. El arte permite crear esquemas mentales que logran una unidad sana, segura y estable entre los niños/jóvenes, madre presa y tutores logrando en ellos cierta autonomía.

En fin, redescubrir lo que vales a través del arte no es un espejismo, es una realidad que ayuda a transfigurar la vida de todo aquel que se encuentre en un estado vulnerable.

\section{Referencias}

Adalist-Estrin, A. (2003). Why maintain relationships. Children of Prisoners Library: Facts and Issues. Recuperado de http://nrccfi.camden.rutgers.edu/files/ cipl102-whymaintainrelationships.pdf

Aporta. (2017). Instituto Renace. Recuperado de https://www.aporta.org.mx/renace/renace-ninios

Balladares, S. y Saiz, M. (2015). Sentimiento y afecto. Ciencias Psicológicas, Mayo, 63-71. Recuperado de http://www.redalyc.org

Cobos, E. G. (2008). Adolescencia y familia: revisión de la relación y la comunicación como factores de riesgo o protección. Revista intercontinental de psicología y educación, 10(2), 105-122. Recuperado de http://www.redalyc.org/pdf/802/80212387006.pdf

Instituto Nacional de Estadística y Geografía. (2017). Documentos de análisis y estadísticas. en Números, Recuperado de http://www.cdeunodc.inegi.org.mx/ unodc/wp-content/uploads/2018/01/en_numeros2.pdf

López, R, B. (2004). Arte terapia. Otra forma de curar. Educación y futuro. Revista de investigación aplicada y experiencias educativas, (10), 101-110. Recuperado de https://dialnet.unirioja.es/servlet/ articulo?codigo $=2044648$

Macías, M. A., Marín, A. P., \& Cantillo, K. V. (2004). Relaciones familiares en familias desplazadas por la violencia ubicadas en" La Cangrejera" (corregimiento de Barranquilla, Colombia). Psicología desde el Caribe, (14). Recuperado de http://www.redalyc.org/ pdf/213/21301405.pdf 
Martí, J. Obras Completas. Editorial de Ciencias Sociales del Instituto Cubano del Libro Tomo 8, P. 291. "Escuela de electricidad" La América. Nueva York, noviembre de 1883.

Medigraphic. (2005). II. Conceptos básicos para el estudio de las familias. Archivos en Medicina Familiar: Vol.7 Supl. 1 Recuperado de www.medigraphic.com/ pdfs/medfam/amf-2005/amfs051c.pdf

Newmark, Gerald. (2002). “Cómo criar niños emocionalmente sanos". Editorial El Manual Moderno. México.

Renace. (2016). Programas Renace. Recuperado de 2017 de http://renace.org.mx/servicios-de-la-comunidad

Sánchez, P. V. (2012). La experiencia vinculante afectiva del sujeto adolescente infractor. Revista Latinoamericana de Ciencias Sociales, Niñez y Juventud, Enero-Junio, pp. 453-465. 